\title{
Diagnostic value of skin RT-QuIC in Parkinson's disease: a two-laboratory study
}

Anastasia Kuzkina (iD) ${ }^{1}$, Connor Bargar ${ }^{2}$, Daniela Schmitt ${ }^{1}$, Jonas Rößle ${ }^{1}$, Wen Wang ${ }^{2}$, Anna-Lena Schubert ${ }^{1}$, Curtis Tatsuoka ${ }^{3,4}$, Steven A. Gunzler $\mathbb{D}^{3,4}$, Wen-Quan Zou ${ }^{2,3}$, Jens Volkmann ${ }^{1}$, Claudia Sommer ${ }^{1}$, Kathrin Doppler $\mathbb{D}^{1,5 \bowtie}$ and Shu G. Chen $\mathbb{D}^{2,3,5 凶}$

Skin a-synuclein deposition is considered a potential biomarker for Parkinson's disease (PD). Real-time quaking-induced conversion (RT-QuIC) is a novel, ultrasensitive, and efficient seeding assay that enables the detection of minute amounts of a-synuclein aggregates. We aimed to determine the diagnostic accuracy, reliability, and reproducibility of a-synuclein RT-QulC assay of skin biopsy for diagnosing PD and to explore its correlation with clinical markers of PD in a two-center inter-laboratory comparison study. Patients with clinically diagnosed PD $(n=34)$, as well as control subjects $(n=30)$, underwent skin punch biopsy at multiple sites (neck, lower back, thigh, and lower leg). The skin biopsy samples (198 in total) were divided in half to be analyzed by RT-QulC assay in two independent laboratories. The a-synuclein RT-QuIC assay of multiple skin biopsies supported the clinical diagnosis of PD with a diagnostic accuracy of $88.9 \%$ and showed a high degree of inter-rater agreement between the two laboratories (92.2\%). Higher a-synuclein seeding activity in RT-QulC was shown in patients with longer disease duration and more advanced disease stage and correlated with the presence of REM sleep behavior disorder, cognitive impairment, and constipation. The a-synuclein RT-QulC assay of minimally invasive skin punch biopsy is a reliable and reproducible biomarker for Parkinson's disease. Moreover, asynuclein RT-QuIC seeding activity in the skin may serve as a potential indicator of progression as it correlates with the disease stage and certain non-motor symptoms.

npj Parkinson's Disease (2021)7:99; https://doi.org/10.1038/s41531-021-00242-2

\section{INTRODUCTION}

Clinical diagnosis of idiopathic Parkinson's disease (PD) is primarily based on the presence of a parkinsonian syndrome along with supportive signs and absence of red flags suggestive of an alternate diagnosis ${ }^{1}$. The wide range of clinical signs and their evolution during the disease course complicate the early and differential diagnosis. Retrospective neuropathological studies have found a highly variable accuracy of clinical diagnosis between 46 and $95 \%^{2}$. As understanding of the disease evolves, additional objective diagnostic markers, such as nuclear brain imaging, have been incorporated into the diagnostic criteria. However, due to the lack of standardization of feasible and easily reproducible assays, tissue biomarkers have not yet been included.

Aggregation of a-synuclein (a-syn) is considered the histoneuropathological hallmark of PD. In the past decade, depositions of asyn have been observed premortally in peripheral nerves in a variety of tissues, including skin that is easily accessible ${ }^{3-5}$. Several studies reported immunohistochemical (IHC) detection of phosphorylated a-syn in dermal nerve fibers ${ }^{5-8}$. However, in spite of numerous follow-up studies that included different protocols for detection of a-syn aggregates and oligomers by IHC and proximity ligation assay, the method has not entered clinical practice ${ }^{7-13}$. Immunohistochemical detection of dermal a-syn is time-consuming, quantification is difficult, and the extent of deposits does not correlate with disease progression ${ }^{5,6}$. A more practical and validated method that allows for rapid analysis of a large number of samples is needed. Ideally, quantification of this biomarker should correlate with disease progression.
Real-time quaking-induced conversion (RT-QuIC) assay, based on prion-like seeding activity of a-syn, has already shown promise in detecting a-syn aggregates in the brain and cerebrospinal fluid (CSF) of patients with synucleinopathies with high accuracy ${ }^{14,15}$. A recent study with skin-based RT-QulC and protein misfolding cyclic amplification assays provided the proof-of-concept evidence that skin a-syn aggregation seeding activity could be a novel biomarker for PD, but data on the validation of the method and its correlation with clinical measures of PD are lacking ${ }^{16}$.

We therefore conducted a structured two-center study to assess the inter-laboratory reliability and reproducibility of the skin RTQulC assay for the diagnosis of PD and to examine its correlation with symptoms and disease progression in order to explore its potential use as a peripheral tissue biomarker.

\section{RESULTS}

\section{Patients and controls}

Thirty-four patients with PD and 30 controls were included in this prospective study. A total of 117 individual biopsies from 34 PD patients as well as 81 biopsies from 30 control subjects were collected. Age and gender did not differ between the PD and control groups. Demographic and clinical data are summarized in Table 1.

\section{a-Syn seeding activity detected by RT-QuIC is increased in skin biopsies from PD patients}

We performed RT-QuIC assay of multiple skin biopsies per subject, from subjects with PD and controls, in a blinded prospective

\footnotetext{
${ }^{1}$ Department of Neurology, University Hospital of Würzburg, Würzburg, Germany. ${ }^{2}$ Department of Pathology, Case Western Reserve University School of Medicine, Cleveland, OH, USA. ${ }^{3}$ Department of Neurology, Case Western Reserve University School of Medicine, Cleveland, OH, USA. ${ }^{4}$ Department of Neurology, University Hospitals Cleveland Medical

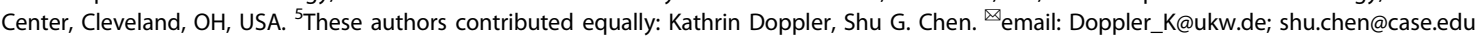


Table 1. Demographic and clinical data of patients and controls.

\begin{tabular}{|c|c|c|c|}
\hline Characteristic & $\mathrm{PD}(n=34)$ & $\begin{array}{l}\text { Controls }(n= \\
30)\end{array}$ & $p$ value \\
\hline Age (years), mean $\pm S D$ & $67.1 \pm 8.6$ & $63.5 \pm 7.9$ & 0.08 \\
\hline Male:female, $n$ & $24: 10$ & $17: 13$ & 0.53 \\
\hline $\begin{array}{l}\text { Disease duration (years), mean } \\
\pm \mathrm{SD}\end{array}$ & $11.7 \pm 6.9$ & NA & NA \\
\hline $\begin{array}{l}\text { Age at diagnosis (years), mean } \\
\pm \text { SD }\end{array}$ & $55.4 \pm 12$ & NA & NA \\
\hline $\begin{array}{l}\text { Hoehn und Yahr stage, mean } \pm \\
\text { SD }\end{array}$ & $2.5 \pm 0.8$ & NA & NA \\
\hline NMSS score sum, mean \pm SD & $46.26 \pm 33$ & NA & NA \\
\hline $\mathrm{MCl}, n(\%)$ & $11(33 \%)$ & NA & NA \\
\hline DBS surgery, $n(\%)$ & $17(50 \%)$ & NA & NA \\
\hline $\begin{array}{l}\text { RBD, positive screening } \\
\text { question, } n(\%)\end{array}$ & $20(59 \%)$ & $2(6.7 \%)$ & $<0.001$ \\
\hline
\end{tabular}

$S D$ standard deviation, $P D$ Parkinson's disease, NMSS Non-Motor Symptom Scale, $M C I$ mild cognitive impairment, $D B S$ deep brain stimulation, $R B D$ REM sleep behavior disorder, NA not applicable.

study. As exemplified in Fig. 1a, RT-QulC assay readily detected asyn seeding activity as shown by the enhanced ThT fluorescence in all four skin biopsies collected from a PD patient but not in those from a control subject. Overall, RT-QulC analyses of the whole cohort confirmed a progressive increase of ThT fluorescence of skin biopsies over time in the PD group $(n=34)$, crossing the cut-off threshold at a mean of $18 \mathrm{~h}( \pm 5)$ and reaching $50 \%$ of the maximum fluorescence at $26 \mathrm{~h}( \pm 5)$, while ThT fluorescence of skin biopsies of the control group $(n=30)$ remained much lower (Fig. 1b, Cleveland data). Accordingly, the final ThT fluorescence intensities $(50 \mathrm{~h})$ were significantly higher in the PD group $(47.2 \pm$ $24.5 \%$ ) than in the control group (7.92 $\pm 9.87 \%), p<0.001$, Fig. 1c). The calculated skin RT-QulC scores of combined data from multiple skin sites of each participant were markedly higher in the PD group compared to the control group ( $p<0.001$, Fig. 1d and Table 2), reflecting significantly higher overall skin a-syn seeding activity in PD.

Based on the cut-off of the skin RT-QulC, 28 PD patients (82.4\%) were classified as positive, $3(8.8 \%)$ as intermediate (Fig. $1 \mathrm{~d})$. In the control group, only 3 patients (10\%) were classified as positive, 1 as intermediate (3.3\%). This translated to an $88.9 \%$ diagnostic accuracy (sensitivity $90.9 \%$, specificity $86.7 \%$ ) if intermediate results were considered equal to positive. When intermediate results were considered as negative, the resulting diagnostic accuracy was lowered to $85.7 \%$ with decreased sensitivity $(81.8 \%)$, but slightly higher specificity $(90 \%)$. A post hoc receiver operating characteristic analysis revealed an optimal skin RT-QulC score cutoff at 0.3 showing a maximum $90.0 \%$ specificity and $91.2 \%$ sensitivity (Fig. 1e), with $31 / 34$ positive PD patients, and $27 / 30$ controls remaining negative. Combined results of two sites (C7 and thigh) reached $85 \%$ sensitivity, while showing an even superior (93\%) specificity (as omitting a supposedly false-positive Th10 site changed the final result of subject CTL30 to negative).

ThT fluorescence induced by skin biopsies from PD patients was higher and occurred more frequently across all anatomical sites (C7, Th10, thigh) as compared to the control subjects $(p<0.001$, Table 2 and Fig. 2a-c). Supporting extensive RT-QulC positivity in PD, skin RT-QulC scores were significantly higher in the PD group than in the control group in all sites (Fig. 2d-f and Table 2, $p<$ $0.001)$, with the best separation between the PD and control group in C7 (Fig. 2d). In positive PD patients $(n=28)$, RT-QulC positivity was generally conserved across multiple biopsy sites (Fig. 3), 19 patients (68\%) were positive at all sites, while all 28 patients (100\%) had at least two positive sites. In contrast, RT-QuIC positivity was only sparsely observed in $3 / 30$ control subjects $(10 \%)$ at individual sites.

\section{High inter-laboratory reproducibility of RT-QuIC}

Inter-laboratory agreement of RT-QulC results (positive/negative) was reached in $92.2 \%$ of cases and in $88.9 \%$ of biopsies (see Table 2). To explore the source of discrepancies, such as due to sample differences (different amount of a-syn seeds in the halves of the biopsy specimen or differences in homogenization procedure) or differences in the RT-QulC assay protocols, a subgroup of lysates was exchanged ( $n=126$ (63.6\%) of 198 total). Same-source lysates analyzed at both laboratories confirmed an excellent agreement between the assays $(k=0.84, p<0.001)$. The comparison of RTQulC of skin lysates originating from different halves of the same biopsy showed a substantial reproducibility in both assays $\left(\kappa_{\text {weighted }}=0.7, p<0.001\right)$.

\section{Clinical characteristics in patients with low a-syn seeding activity and in controls with positive RT-QuIC scores}

PD patients with negative $(n=3)$ and intermediate $(n=3)$ RTQulC results were re-evaluated, but no red flags indicating an alternative diagnosis were found. All but one of the patients fulfilled the International Parkinson and Movement Disorder Society (MDS) criteria for a clinically established PD. Notably all 6 patients had a normal Montreal cognitive assessment (MoCA) and a significantly lower burden of non-motor symptoms (NMSs) (Non-Motor Symptoms Scale (NMSS) scores of 31.5 (9.38) compared to 49.4 (35.5) in positive patients, $p=0.027$ ). In the RT-QulC-negative patient with probable PD, only one supportive criterion (response to levodopa) could be identified, while rest tremor, dyskinesia, and olfactory loss (verified by normal Sniffin' sticks assessment) were lacking, and disease duration was just 1 year. In one of the intermediate cases, a bloody lysate appearance was documented, possibly leading to a false-negative result ${ }^{17}$.

Among the three control subjects with a positive RT-QulC score, one (CTL30; Fig. 3 and Supplementary Table 1) had olfactory dysfunction (anosmia on Sniffin' sticks assessment) and possible rapid eye movement sleep behavior disorder (RBD) as reported in the single-question screening. The second control subject (CTL13) reported RBD. The third control person (CTL9) reported no signs suggesting prodromal PD apart from urinary dysfunction, depression, and idiopathic small fiber neuropathy. Notably, in the latter case only the lysate from one of the halves of the biopsy tested positive in both Würzburg and Cleveland laboratories, whereas the other half remained negative.

\section{a-Syn seeding activity in skin biopsies correlates with disease duration and stage}

Disease duration positively correlated with the final percentage of ThT fluorescence $(r=0.4, p=0.02)$ and negatively correlated with T50 (time to reach half maximum fluorescence, $r=-0.36, p=$ 0.038 ) and lag phase (time to cross threshold fluorescence, $r=$ $-0.38, p=0.025$ ), as demonstrated in Fig. 4a, $c, f$ (data from Cleveland center shown). Moreover, Hoehn and Yahr (H\&Y) stage moderately correlated with the final percentage of ThT fluorescence ( $r=0.46, p=0.0068$, Fig. $4 d$, Cleveland center). Finally, the skin RT-QulC score as an integrated measure of a-syn seeding activity of both Cleveland and Würzburg centers also positively correlated with both the disease duration $(\rho=0.37, p=0.032)$ and the H\&Y stage $(\rho=0.39, p=0.024$, Fig. $4 \mathrm{~b}$, e).

To further evaluate whether a longer disease duration was associated with a higher burden of peripheral a-syn aggregates, Spearman-Kärber analysis of endpoint dilutions was utilized to determine the seeding dose $\left(\mathrm{SD}_{50}\right)$ at which $50 \%$ of replicate 

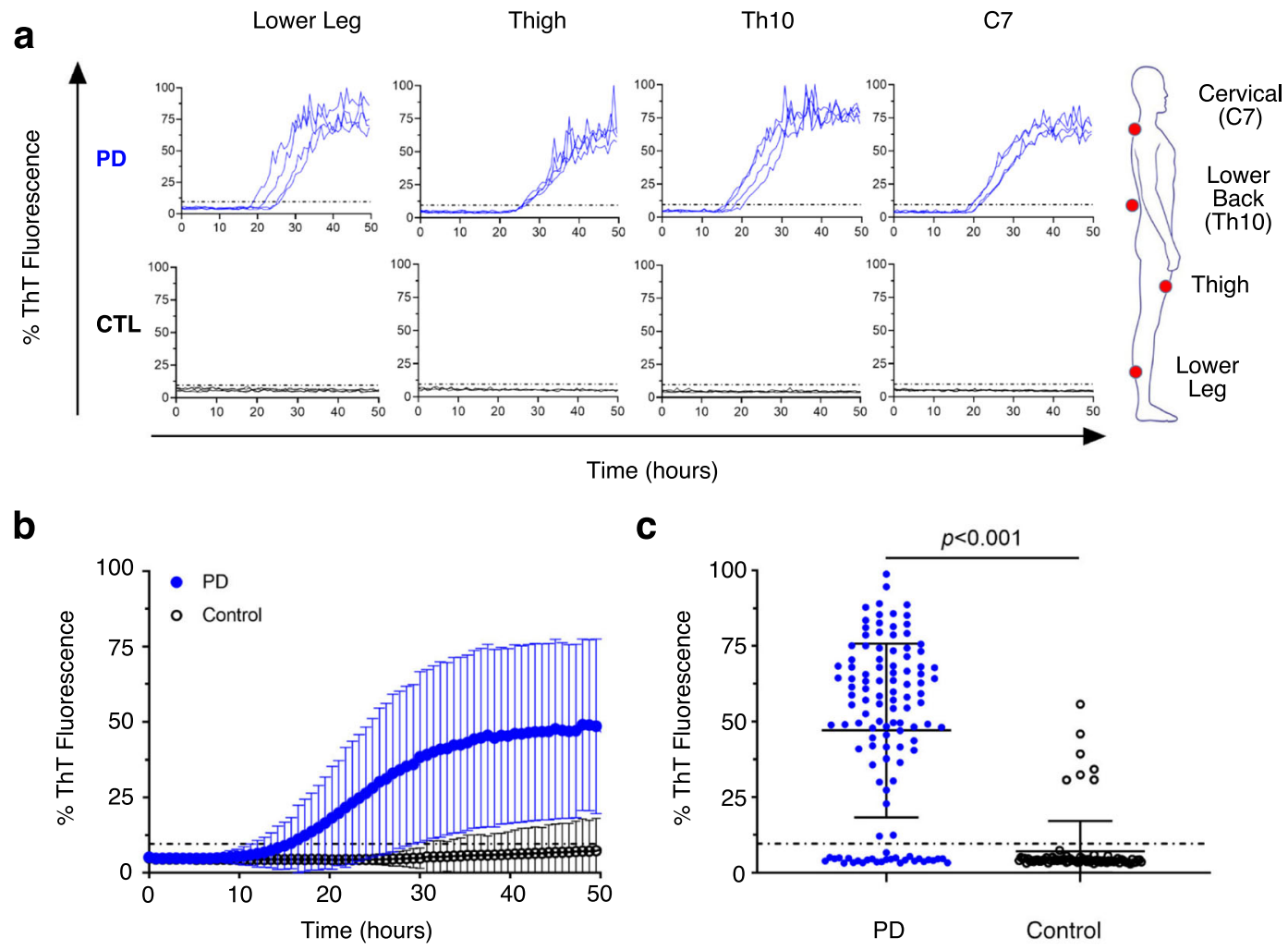

d

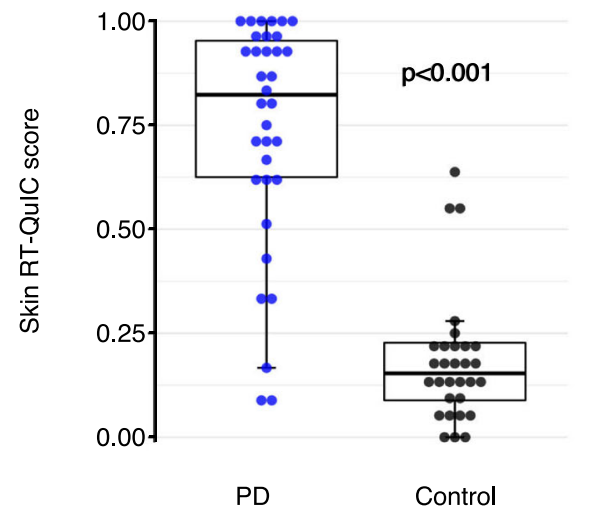

e

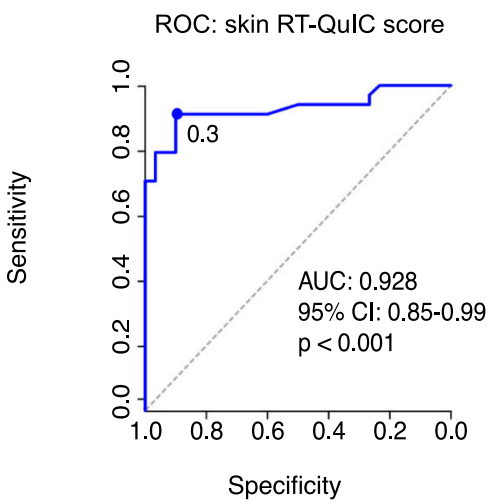

Fig. 1 RT-QulC detection of a-syn seeding activity in skin biopsies from PD patients and control subjects. a $\alpha$-Syn RT-QulC assay of multiple skin biopsies from a PD patient (PD10) and a control subject (CTL2). Four skin biopsy sites at the level of C7 (neck), Th10 (lower back), thigh, and lower leg are indicated. Shown are individual ThT fluorescence responses over time for each skin sample tested in quadruplicate. The dotted line indicates threshold at $9.6 \%$. A marked increase of ThT fluorescence over time is found in patient samples but not in the control samples. b Cumulative results of blinded testing of all skin biopsies by $\alpha$-syn RT-QulC (Cleveland center). Mean values ( \pm SD) of percentage of ThT fluorescence of all biopsies tested in quadruplicate are clearly increased in PD patients $(n=34)$ compared to control subjects $(n=30)$. The dotted line indicates threshold at $9.6 \%$. c Scatter graph of final percentage of ThT fluorescence intensities $(50 \mathrm{~h})$ for all individual biopsies. Average ThT fluorescence intensities of skin biopsies from PD patients $(n=34$ with 117 biopsies) are significantly higher $(p<0.001)$ than those from control subjects ( $n=30$ with 81 biopsies). Dots represent values of individual biopsies, the dotted line indicates threshold at $9.6 \%$. Group mean \pm SD are represented by solid lines. $\mathbf{d}$ Skin RT-QulC scores in PD patients $(n=34)$ and control subjects $(n=30)$. Scores are pooled from both Cleveland and Würzburg centers. A box plot demonstrates significantly higher scores in PD compared to controls $(p<0.001)$. The horizontal line in the box plot marks the median, the box represents the interquartile range (IQR), and the whiskers extend to the most extreme data point, which is no more than 1.5 times of IQR. e Receiver operating characteristic (ROC) curve and the corresponding area under the curve (AUC) for skin RT-QulC scores. ROC analysis demonstrates an optimal RT-QulC score threshold of 0.3 that would result in a $91.2 \%$ sensitivity and $90.0 \%$ specificity. $\mathrm{Cl}$ confidence interval.

reactions were positive. This allowed for a quantitative comparison of a-syn seeding activity (in $\mathrm{SD}_{50}$ units) among specimens from different patients, similar to the measures used in bioassays ${ }^{18}$. Serial dilutions of skin biopsies at C7, Th10, and thigh from PD patients with shorter ( $5-7$ years, $n=3$ ) and longer (15-28 years, $n$ $=4$ ) disease duration were performed. Parallel comparisons revealed that a-syn seeding activity persisted in more diluted skin lysates in patients with a long disease duration as compared to those with a short duration (Fig. $5 \mathrm{a}$ ), with $\mathrm{SD}_{50}$ values being fourfold higher in the long duration patients (Fig. 5b; median $\mathrm{SD}_{50} / \mathrm{mg}, 2430$ units for long duration vs 616 units for short duration; $p=0.0083$ ). 


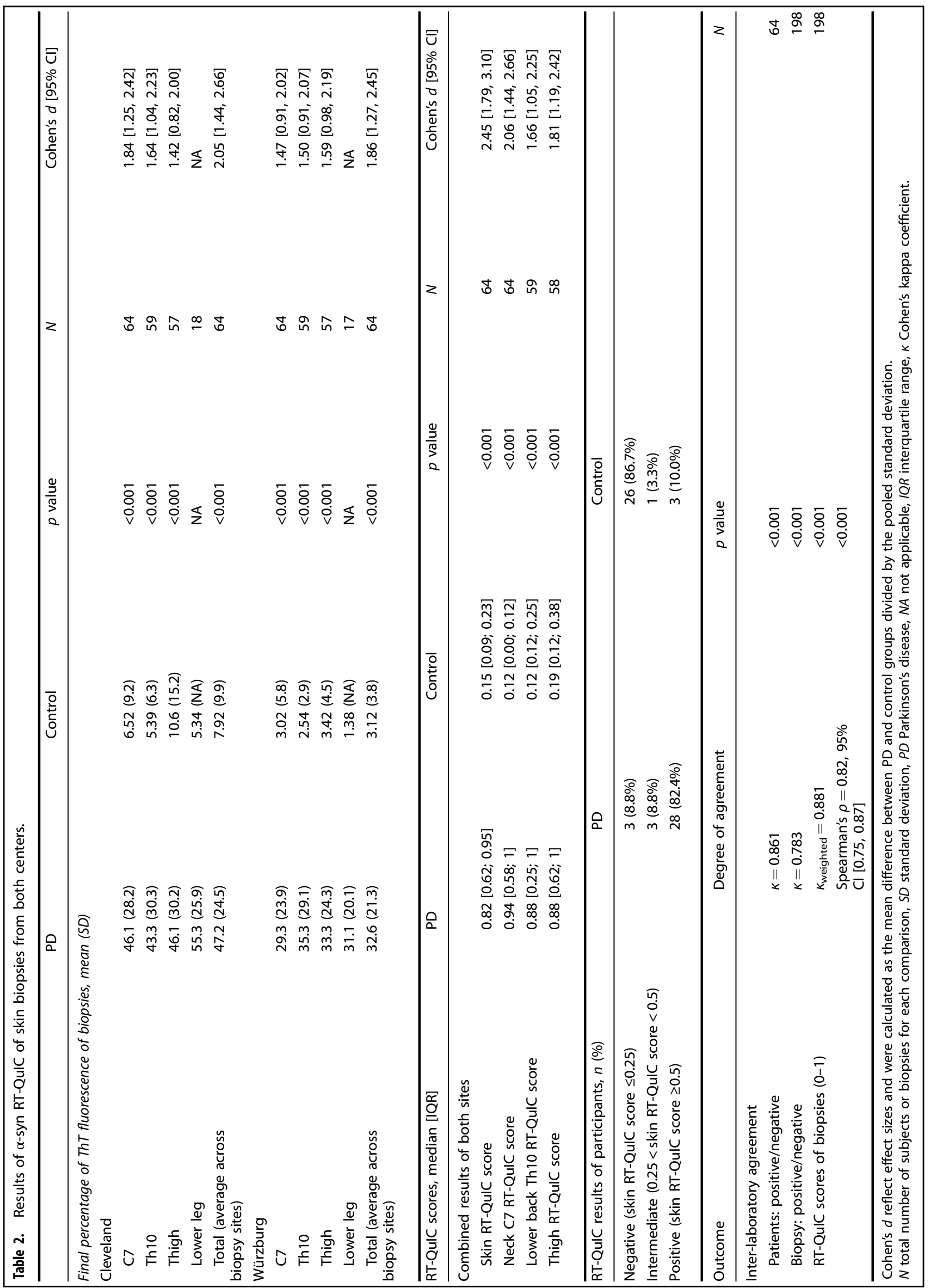




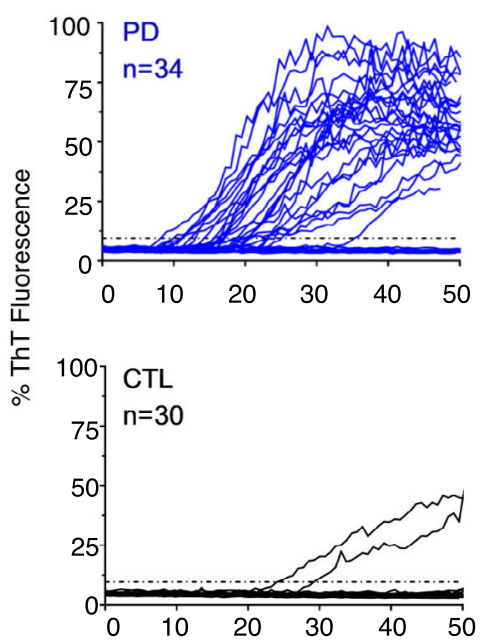

d

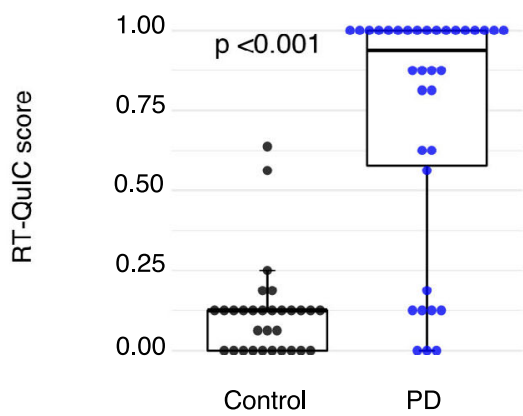

b
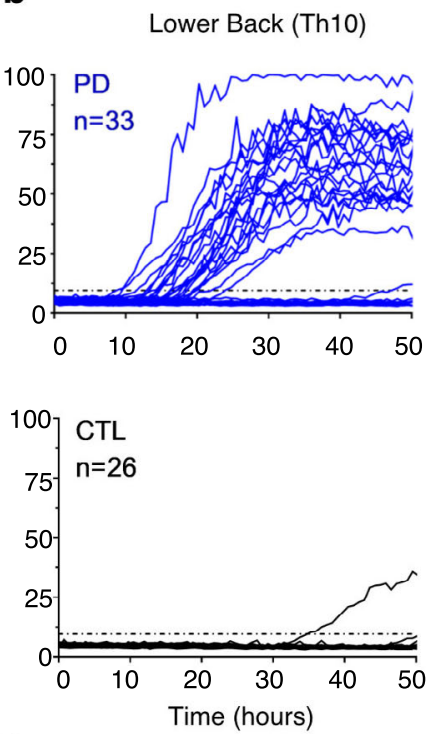

e

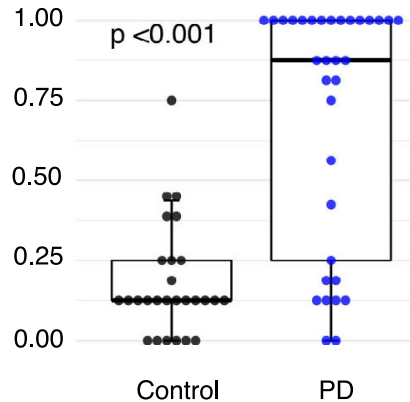

C
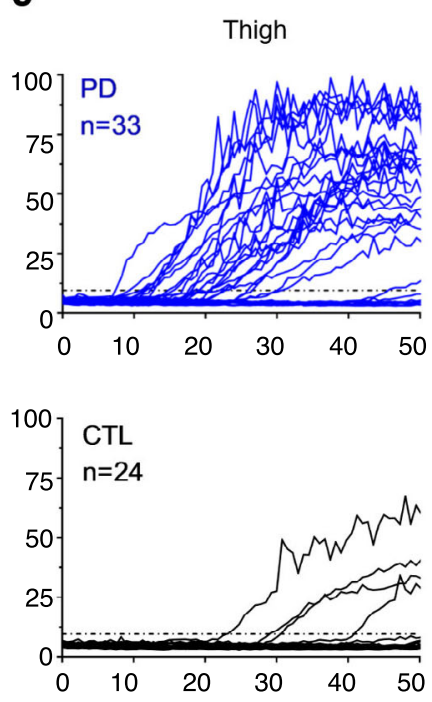

f

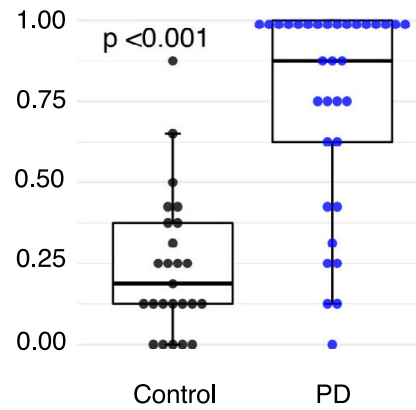

Fig. 2 Higher a-syn seeding activity in skin biopsies from different anatomical sites in PD patients as compared to control subjects. a-c show RT-QulC reactivity in skin biopsies of PD patients and controls collected at the locations of cervical C7, lower back Th10, and thigh, respectively. ThT fluorescence from individual skin biopsies is more intense and occurs more frequently in PD (blue curves) than in controls (CTL, black curves) above the threshold of $9.6 \%$ (dotted lines). Raw data are taken from the Cleveland center. d-f illustrate the RT-QulC scores for individual skin biopsies in PD and control groups collected at cervical C7 (d), thoracal Th10 (e), and thigh (f). Scores are pooled from both Cleveland and Würzburg centers. The box plots demonstrate significantly higher scores in PD patients compared to controls $(p<0.001)$. The boxes represent the interquartile range (IQR) with the median line and the whiskers extending to the most extreme data point, which is no more than 1.5 times of IQR.

\section{Higher a-syn seeding activity is found in patients with certain NMSs}

The skin RT-QulC score was higher in PD patients who reported $\operatorname{RBD}(0.93[0.8 ; 0.98]$ vs $0.63[0.32 ; 0.75], p<0.01)$, constipation $(0.94[0.89 ; 0.97]$ vs $0.65[0.41 ; 0.8], p<0.01)$, and in patients with mild cognitive impairment $(\mathrm{MCl})(0.98[0.93 ; 1]$ vs $0.73[0.45 ; 0.87])$, $p<0.01$ ) (Fig. 6a-c). The MoCA score negatively correlated with the skin RT-QulC score $(\rho=-0.56, p=0.0014$, Fig. $6 \mathrm{~d})$. Skin RTQulC score was not significantly higher in patients who reported hyposmia on NMSS (Fig. 6e). While the correlation between the skin RT-QulC score and total NMSS score was not significant, the number of reported key NMSs (orthostatic dysfunction, restless legs syndrome, constipation, urinary dysfunction, hyposmia, RBD, insomnia, day-time sleepiness, depression, and $\mathrm{MCl}$ ) positively correlated with the skin RT-QulC score $(\rho=0.4, p=0.02$, Fig. 6f). We did not find a significant difference in skin RT-QulC score between motor subtypes of PD (the tremor-dominant PD $(0.78$ $[0.62 ; 0.86]$ vs the akinetic-rigid PD $(0.86[0.67 ; 0.97], p=0.705)$.

\section{DISCUSSION}

We conducted a dual-center comparison study of a skin RT-QulC assay, demonstrating excellent diagnostic accuracy and inter- laboratory reproducibility for detection of skin a-syn seeding activity in PD. RT-QulC results also correlated with progression markers and NMSs of PD, suggesting that RT-QulC has potential value as a biomarker in $\mathrm{PD}$.

The diagnostic accuracy in our study was up to $88.9 \%$ with sensitivity of $81.8-90.9 \%$ and specificity of $86.7-90 \%$ (depending upon whether intermediate results were counted as positive or negative). The overall higher sensitivity compared to prior IHC studies likely indicates that RT-QulC can detect IHC undetectable a-syn aggregates ${ }^{5,9,19}$. A side-to-side comparison with IHC could provide a more reliable comparison of the methods as well as some insight into the type of affected fibers, which is known to differ between synucleinopathy subtypes ${ }^{20-22}$ and should be addressed in future studies. Moreover, RT-QulC assay results were highly reproducible in our inter-laboratory comparison compared to IHC, where sensitivity of phosphorylated a-syn detection varied widely between groups ${ }^{6,23}$. Some minor discrepancies in the results were present, possibly due to slight differences in the RTQulC assay protocols.

Skin a-syn seeding activity was nearly ubiquitously present in PD. The most proximal (C7) site showed a slightly superior accuracy, similar to prior studies in $\mathrm{IHC}^{5,6}$. However, likely due to an overall higher sensitivity of RT-QulC, we did not find a striking 
a

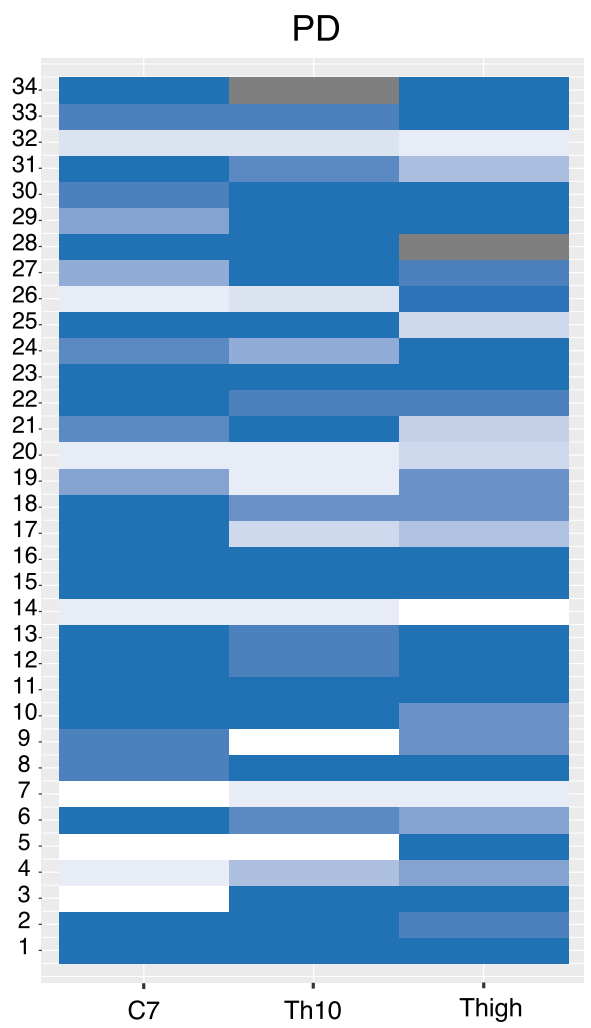

Total positive, $\mathrm{n}(\%)$ b

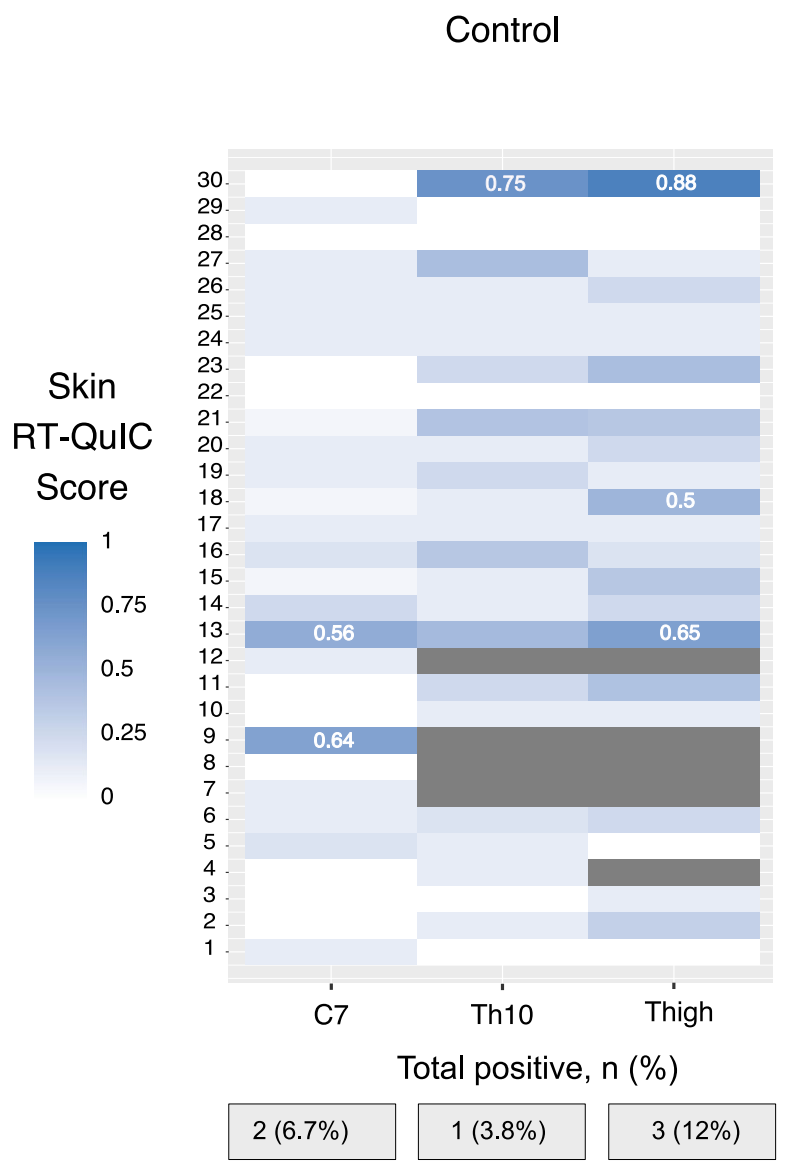

Fig. 3 a-syn seeding activity is conserved across multiple biopsy sites in PD and is universally low in controls. Distribution of skin RT-QulC scores ranging from 0 (white) to 1 (blue) across cervical (C7), thoracal (Th10), and thigh sites in PD patients (a) and control subjects (b). The graphs show a mostly uniform presence of skin $\alpha$-syn seeding activity in individual biopsy sites in PD patients, and only sparsely in single biopsy sites in controls. Subjects are listed vertically. The total number (\%) of biopsies with positive RT-QuIC scores ( $\geq 0.5)$ at each site for the PD and control groups is indicated below each graph. Missing biopsy spots are shaded gray. Scores are pooled from both Cleveland and Würzburg centers.

difference in positivity of proximal to distal sites. The lower back site (Th10) was slightly less sensitive, probably due to higher thickness of derma that complicates the separation of the biopsy punch ultimately leading to a smaller biopsy size. Sensitivity of each of the sites in isolation was still substantially inferior to the evaluation of all sites combined. The need to perform multiple punches to reach high diagnostic certainty is therefore a limitation of this approach. However, retrospectively, combined results of the two sites that showed highest accuracy (C7 and thigh) still allowed to reach $85 \%$ sensitivity and $93 \%$ specificity. The diagnostic accuracy from this relatively small trial should be validated in a larger cohort.

The skin a-syn RT-QulC seeding activity moderately correlated with H\&Y stage and duration of disease. However, the correlation is limited by low number of patients in advanced disease stages and might have biased the lower diagnostic certainty in patients with short disease duration. A correlation of skin a-syn pathology with the H\&Y stage was previously reported in a subset of IHC trials using highly diluted primary antibodies against total a$\operatorname{syn}^{10,11}$, possibly causing a preferential binding to the aggregated over the physiological a-syn ${ }^{9,24}$. IHC studies that used antibodies against the phosphorylated form of a-syn could not reproduce this finding ${ }^{5,6}$. However, detection of dermal a-syn aggregates by $\mathrm{IHC}$ may be biased by the patchy distribution of a-syn aggregates in the skin resulting in sampling errors, as only a limited number of skin sections can be analyzed. In contrast, RT-QulC allows for the analysis of whole-biopsy lysates. One of the a-syn seeding studies of $\mathrm{CSF}^{25}$ found a correlation with H\&Y stage, while others could not confirm $i^{26,27}$. Interestingly, our serial endpoint dilution analyses revealed that skin biopsies in PD patients with longer disease duration had higher RT-QulC seeding units than those with shorter duration, suggesting enhanced accumulation of aggregated a-syn in peripheral tissues during protracted disease course and potential use of skin RT-QulC to monitor progression. The correlations with disease progression were moderate and ultimately prospective studies with biopsy assessments at multiple time points would be needed to further validate a correlation with the disease stage and duration.

Clinical relevance of skin RT-QulC is further supported by the correlation of the skin RT-QulC score with NMSs. Dermal a-syn deposition in PD is known to primarily affect the autonomic nervous system ${ }^{5,20,22}$ and was previously shown to be associated with dysautonomia ${ }^{10}$. In a recent study, peripheral a-syn deposition assessed in the gastrointestinal system and skin both correlated with Lewy body stage in the brain ${ }^{28}$. Therefore, involvement of different parts of the peripheral nervous system possibly occurs in parallel, and this could explain correlation of constipation with skin a-syn aggregation in our study. RBD is associated with early dermal a-syn deposition detectable by IHC, as it has been found in more than half of prodromal RBD patients ${ }^{19,29}$. In one study, dermal a-syn deposition in RBD was even higher than in $\mathrm{PD}^{30}$, suggesting a subgroup with a distinct 
a

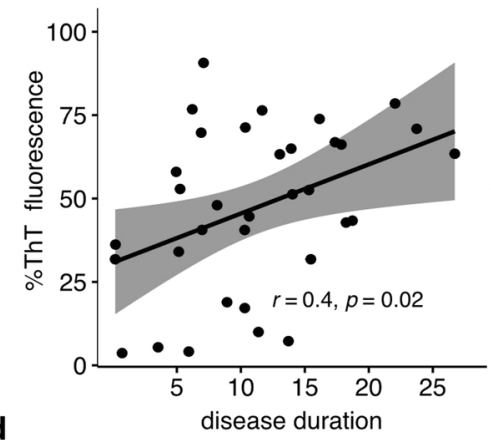

d

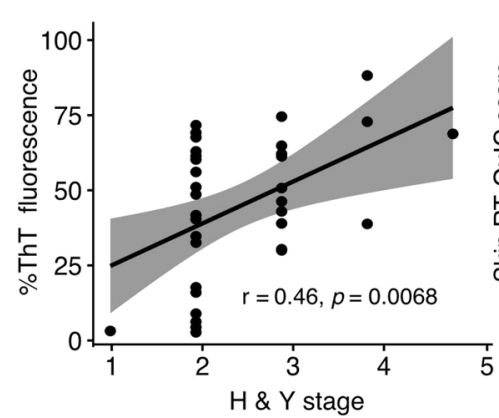

b
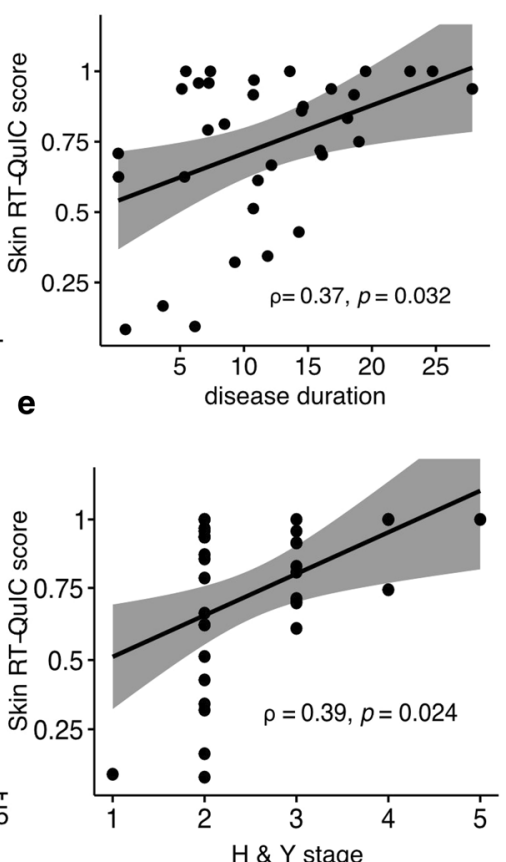

C
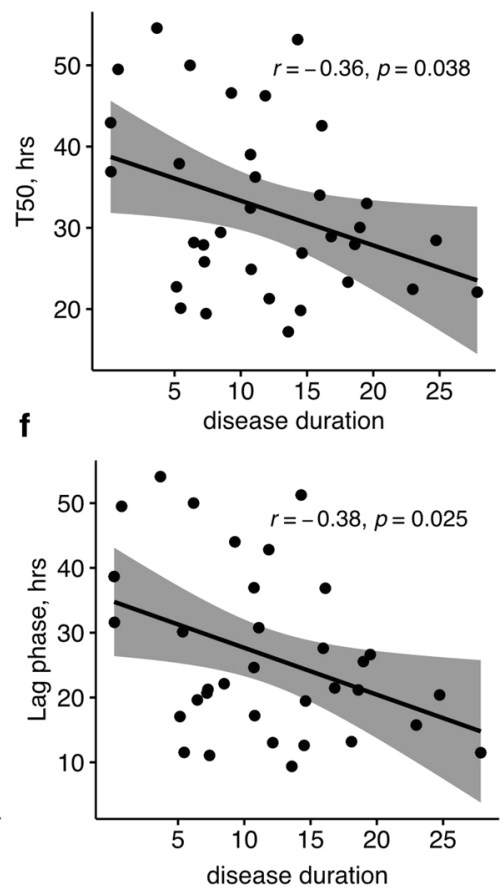

Fig. 4 Skin RT-QuIC results correlate with disease progression. Raw RT-QulC assay parameters correlate with PD duration: longer disease duration is associated with a higher final percentage of ThT fluorescence (a), shorter duration of T50, time needed to reach $50 \%$ aggregation (c), and shorter lag phase (f). d Higher H\&Y stage is associated with a higher final percentage of ThT fluorescence. Raw data (a, $\mathbf{c}, \mathbf{d}, \mathbf{f})$ are taken from the Cleveland assay. b, e Skin RT-QulC scores (0-1) increase with longer disease duration and higher H\&Y stage. Linear regression lines with $95 \%$ confidence interval (gray shade) are shown. Scores are pooled from both Cleveland and Würzburg centers.

disease evolution. Supporting this, PD patients suffering from RBD have been reported to have more $a$-syn lesions in the brain $^{31}$ and higher a-syn RT-QulC seeding activity in $\mathrm{CSF}^{27}$. RBD and constipation are the most common prodromal symptoms of $\mathrm{PD}^{32}$ and are both associated with a faster progression and presence of $\mathrm{MCl}^{33}$. A higher baseline a-syn seeding activity in CSF was recently shown to be predictive of cognitive decline ${ }^{34}$. Similarly, all three non-motor features of RBD, cognitive impairment, and dysautonomia have been shown to be the key determinants of clustering into a "diffuse malignant" phenotype with higher symptom burden and faster disease progression ${ }^{35}$. Correlation of peripheral, in this case dermal, a-syn pathology with "central" symptoms (RBD and $\mathrm{MCl}$ ) further supports the systemic nature of a-syn deposition in both central and peripheral tissues as suggested by pathological studies ${ }^{8,28}$. Higher skin a-syn seeding activity as an indicator of higher peripheral, and possibly overall load of a-syn aggregates, in patients with a "malignant phenotype" may imply a potential use of our skin RT-QulC assay to identify subgroups of PD with different rates of disease progression.

While RT-QuIC supported the clinical diagnosis in most cases, a subgroup of PD patients showed no or low a-syn seeding activity. Only in one of these cases, blood contamination was apparent in the lysates possibly inhibiting aggregation ${ }^{17}$. Moreover, five of these patients reached the highest degree of diagnostic certainty according to MDS criteria, making a false clinical diagnosis unlikely. RT-QulC-negative PD patients may represent a suggested central nervous system-first phenotype ${ }^{36}$, in which the basal ganglia pathology is postulated to appear prior to the involvement of the peripheral nervous system. This is supported by a lower occurrence of NMSs in these patients. Alternatively, these could be patients in whom a-syn pathology is not consistently found, as in some genetic forms with Parkin, PINK1, and LRRK2 mutations ${ }^{37,38}$. Though none of the patients were genetically tested, 2 of the RT-QulC-negative patients had an early-onset disease (at 37 and 42 years), and 1 had a positive family history. Moreover, four out of six patients with negative or intermediate RT-QulC results had prior deep brain stimulation (DBS) surgery, and patients with genetic parkinsonism were previously shown to be overrepresented in the DBS collective due to the predominant motor levodopa-responsive symptoms ${ }^{39}$. Similar to our observations, in a recent CSF RT-QulC study, three PD patients who tested negative exhibited a remarkably mild disease severity ${ }^{40}$. The absence of a-syn seeding activity in the skin may thus suggest lack of involvement of the peripheral nervous system or absence of asyn pathology altogether in a small subgroup of PD patients.

Two control subjects reported possible RBD on screening, and both were positive in RT-QulC, indicating a possible prodromal synucleinopathy. RBD is the most reliable prodromal PD manifestation and is associated with a-syn deposition in dermal autonomic nerves ${ }^{19,29}$. In an earlier CSF study ${ }^{25}$, two of the four control subjects with positive RT-QulC were found to develop PD several years after CSF sampling, supporting the ability of the asyn seeding assay to detect prodromal PD stages more often than older methods, such as IHC. This could have great value for enrollment of prodromal PD subjects in neuroprotective trials. It should be noted that skin RT-QulC scores of control subjects with positive RT-QulC were still markedly lower than the average score of the PD group.

An important limitation of our study that must be addressed in the future is omission of patients with atypical parkinsonism. Skin RT-QulC appears particularly interesting for differential diagnosis with multiple system atrophy, where a-syn is known to preferentially deposit in somatosensory fibers in contrast to autonomic fibers in $\mathrm{PD}^{20-22}$ and was recently shown to be biochemically distinct in a CSF study ${ }^{41}$.

In summary, our study shows that the skin RT-QulC assay achieves a high diagnostic accuracy for PD, similar to the more 


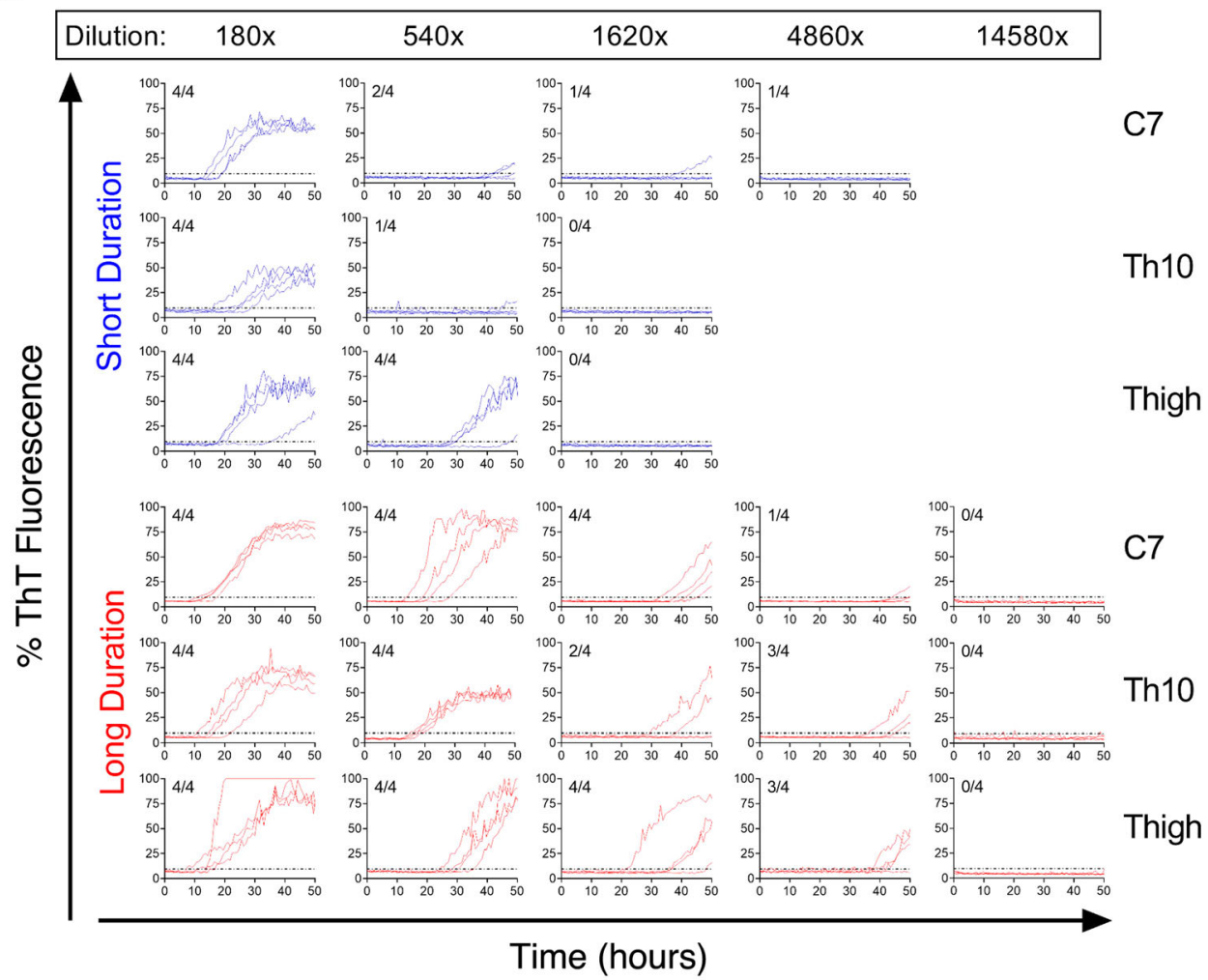

b

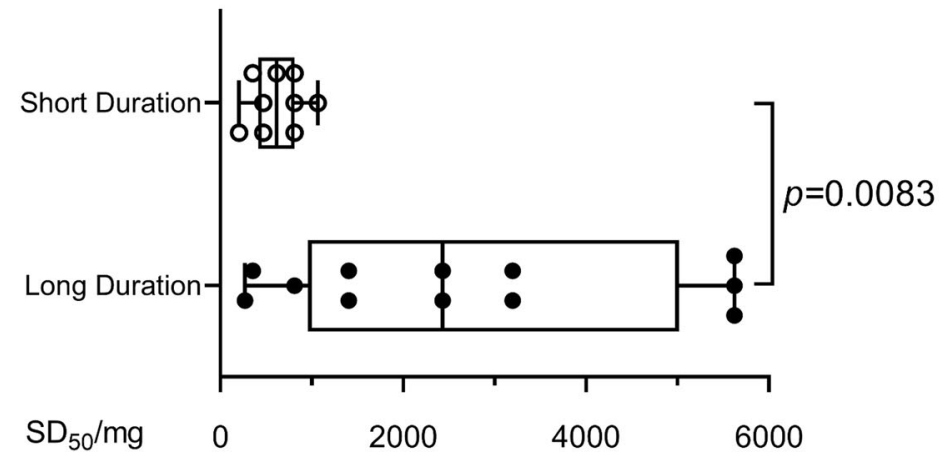

Fig. 5 Comparisons of a-syn seeding activity in skin biopsies of PD patients with different disease duration through endpoint dilution analyses. a RT-QulC spectra of serially endpoint diluted skin biopsies collected at C7, Th10, and thigh from a PD patient with a short (7 years, blue curves) or long (28 years, red curves) disease duration. Dilution folds are indicated on the top. Shown are ThT fluorescence traces from four replicate reactions at the designated dilutions. The fractions in the upper left corner of each graph indicate the positive/total replicate reactions. Dotted lines indicate a threshold of $9.6 \%$. b Values of $\mathrm{SD}_{50} / \mathrm{mg}$ obtained from Spearman-Kärber endpoint analyses for skin biopsies from all anatomic sites of PD patents with short (5-7 years, $n=3)$ or long $(15-28$ years, $n=4)$ disease duration. A box plot demonstrates significantly higher $\mathrm{SD}_{50} / \mathrm{mg}$ in PD patients with long disease duration compared to those with short duration $(p=0.0083$, Mann-Whitney $U$ test). The boxes represent the interquartile range with the median line and the whiskers extending to the most extreme data points.

intensively studied $\operatorname{CSF}^{14,25,26}$. Skin biopsy has the advantage of minimal invasiveness that allows for repeated sampling in longitudinal studies. Our RT-QulC assay was highly reproducible, as illustrated by the two-center inter-laboratory comparison. In addition to the diagnostic value of this assay, the RT-QulC skin assay may offer an opportunity to improve our understanding of the evolution of PD pathogenesis in the peripheral dermal tissue in living patients. We provide evidence for a moderate correlation of skin a-syn RT-QulC seeding activity with disease duration, stage, and certain NMSs. Our promising results warrant further prospective exploration in larger patient populations including early or even prodromal stages of disease. To sum it up, skin biopsy RT-QulC holds great promise as a reliable PD biomarker and may warrant broader implementation in clinical practice as well as usage as an outcome measure in disease-modifying clinical trials.

\section{METHODS}

\section{Study design}

This prospective study was approved by the Ethics Committee of the University of Würzburg. All participants signed informed consent. Patients and controls were recruited at the Department of Neurology, University Hospital Würzburg. Patients with an expert clinical diagnosis of probable or established PD based on the criteria of the MDS were included ${ }^{1}$. The stage of disease was assessed using the H\&Y scale ${ }^{42}$. NMSs were assessed using the German version of the NMSS for $\mathrm{PD}^{43}$. Patients were screened for $\mathrm{MCl}$ using the MoCA and for RBD using a single-question screening ${ }^{44}$. Controls were defined as probands without a history of parkinsonism or dementia 
a

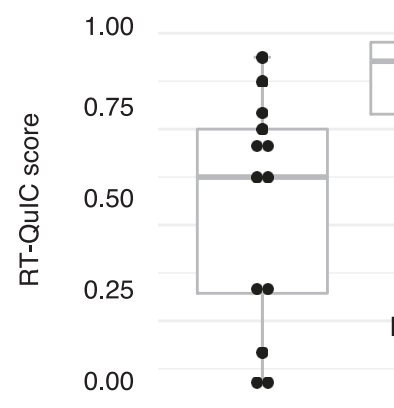

no $\operatorname{RBD}(\mathrm{n}=13) \quad \operatorname{RBD}(\mathrm{n}=20)$

C

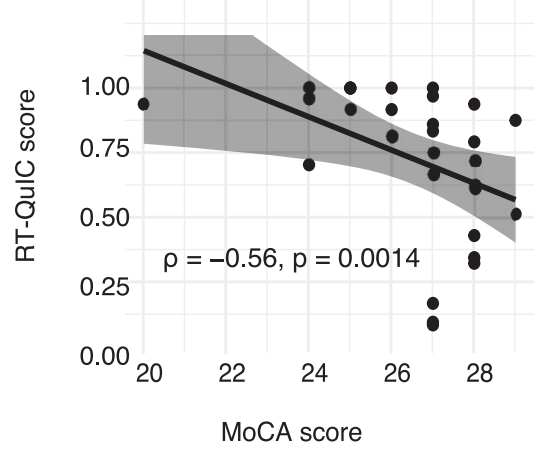

b

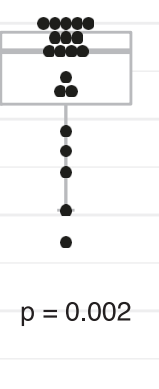

e

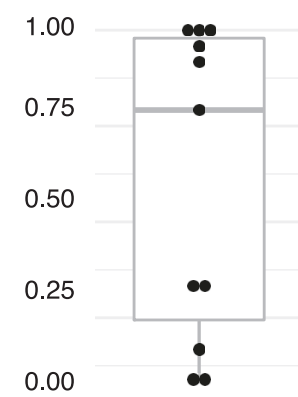

no hyposmia $(n=11)$

0.75

0.50

0.25

0.00

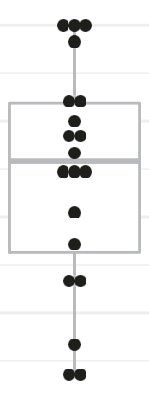

C

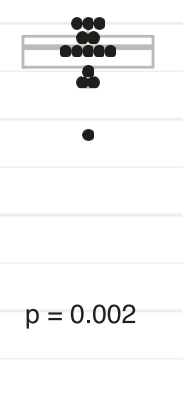

1.00

0.75

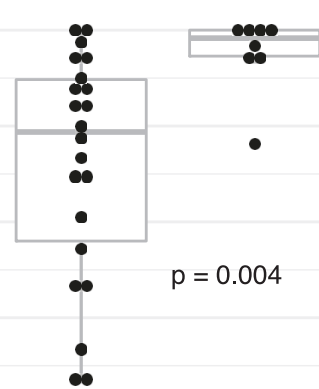

no $\mathrm{MCl}(\mathrm{n}=22)$

$\mathrm{MCl}(\mathrm{n}=8)$

f
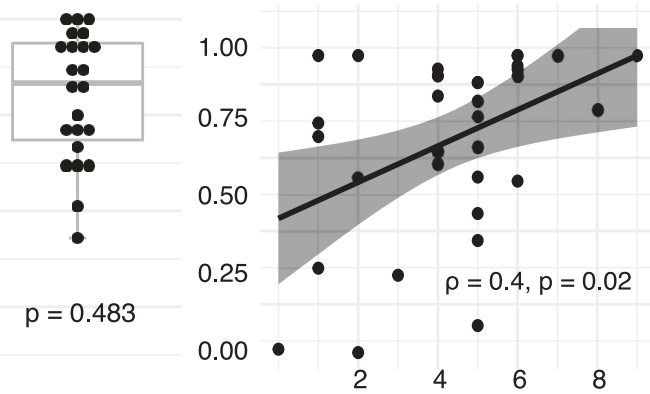

number of reported NMS

Fig. 6 Skin RT-QuIC scores correlate with non-motor symptom burden. a-c, e Box plots illustrating differences in skin RT-QulC scores between subgroups reporting certain non-motor symptoms. Skin RT-QulC scores (vertical axis: 0-1) pooled from both centers are higher in patients with possible RBD (a), constipation (b), and $\mathrm{MCl}(\mathbf{c})$. The skin RT-QulC scores are not significantly higher in patients reporting hyposmia (e). The boxes represent the interquartile range (IQR), the middle line of each box indicates the median value with the median line and the whiskers extending to the most extreme data point, which is no more than 1.5 times of IQR. Values of individual patients are illustrated by dots. d, f Scatter plots illustrating correlations of skin RT-QulC scores with non-motor symptoms. The skin RT-QulC score negatively correlated with MoCA scores (d) and positively with the total number of reported key non-motor symptoms (f), respectively. A linear model regression line is shown, the gray shade represents the $95 \%$ confidence interval.

and showing no signs of parkinsonism on neurological examination. Controls were represented by patients' spouses or recruited from the patient populations treated in the neurology department for unrelated diseases (see Supplementary Table 1) and were assessed using the MDS research criteria for prodromal $\mathrm{PD}^{45}$ and the single-question screening for $\mathrm{RBD}^{44}$.

\section{Skin biopsy procedure}

Skin punch biopsies ( $5 \mathrm{~mm}$ diameter) were performed at locations similar to those described in previous studies ${ }^{5,19}$, namely at C7 and Th10 paraspinally, lateral upper thigh and lower leg (the latter were only performed in a subset of subjects, see Supplementary Table 1). The biopsy specimens were divided into two halves perpendicularly to the epidermis to be processed by both laboratories separately, and both halves were flash-frozen in liquid nitrogen, blindly coded, and stored at $-80^{\circ} \mathrm{C}$.

\section{RT-QuIC assay and lysate preparation}

One half of each skin biopsy was analyzed at the Cleveland center. The RTQulC assay procedure, established based on our published protocol ${ }^{19}$, was modified and optimized for the small skin biopsy. The frozen skin biopsy specimen ( $30 \mathrm{mg}$ wet weight) was thawed and washed in ice-cold phosphate buffer at least three times until no visible blood remained. The skin tissue was cut into tiny pieces on a cold surface, followed by homogenization in 20 volumes of Dulbecco's phosphate-buffered saline (PBS) supplemented with 1\% Triton X-100, $150 \mathrm{mM} \mathrm{NaCl}, 5 \mathrm{mM}$ EDTA, and proteinase inhibitors (Roche mini-cOmplete ${ }^{\mathrm{TM}}$, Roche Diagnostics, USA) with $1 \mathrm{~mm}$ zirconia beads agitated for 5 cycles of $1 \mathrm{~min}$ beating and $3 \mathrm{~min}$ cooling at $4{ }^{\circ} \mathrm{C}$ in a miniBeadbeater-16 device (BioSpec Products, USA). The resulting $5 \%$ skin lysate was further diluted 1:10 with PBS/N2 Gibco supplement (ThermoFisher, USA), from which $2 \mu$ lof the diluted lysate (equivalent to 200-fold dilution of skin tissue [w/v]) was added to the reaction mixture containing $40 \mathrm{mM} \mathrm{NaPO} 4, \mathrm{pH} 8,170 \mathrm{mM} \mathrm{NaCl}, 20 \mu \mathrm{M}$ thioflavin T (ThT), $10 \mu \mathrm{g}$ commercial recombinant a-syn (rPeptide, USA), and 6 silica glass beads (OPS Diagnostics, USA) in a total volume of $100 \mu \mathrm{l}$ per well of a black 96 -well plate with a clear bottom (Nunc, ThermoFisher, USA). Four replicate reactions were made for each skin sample. The plate was sealed with Nunc clear sealing film and incubated at $42^{\circ} \mathrm{C}$ in a BMG FLUOstar Omega plate reader (BMG Labtech, USA) with cycles of $1 \mathrm{~min}$ shaking (400 rpm, double orbital) and 1 min rest throughout the assay. ThT fluorescence (in arbitrary units [AU] at $450 \pm 10 \mathrm{~nm}$ excitation and $480 \pm$ $10 \mathrm{~nm}$ emission; bottom read) was recorded every $45 \mathrm{~min}$ for a total run of $50 \mathrm{~h}$. Raw data were normalized to a percentage of the maximal fluorescence response $(260,000 \mathrm{AU})$. A sample was considered positive when at least two out of four replicates crossed the threshold of background fluorescence plus 5 standard deviations (yielding a 9.6\% cutoff threshold) within $50 \mathrm{~h}$. For each positive sample, the average ThT fluorescence intensity of the positive replicates was plotted against time. For each negative sample, the average fluorescence intensity of the negative replicates was plotted against time. For Spearman-Kärber analyses to obtain the seeding dose giving ThT positivity in $50 \%$ of replicate wells $\left(\mathrm{SD}_{50}\right)$, threefold serial endpoint dilutions of a skin sample were prepared. Each diluted sample was used to seed RT-QulC reactions in quadruplicate. ThT fluorescence from individual replicates for each dilution was plotted against time. $\mathrm{SD}_{50}$ per $\mathrm{mg}$ skin tissue was calculated based on the Spearman-Kärber equations as described ${ }^{18}$.

The other half of each biopsy was assessed at the Würzburg center using a similar assay procedure as described above with some modifications. Briefly, two 7-mm steel beads were used to homogenize skin tissue using the TissueLyser LT device (Qiagen, Germany) to prepare 5\% skin lysates. The lysates were further diluted tenfold and $2 \mu \mathrm{l}$ was added to a reaction

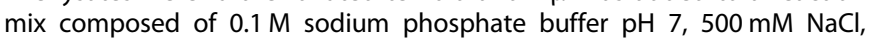


$10 \mu \mathrm{M}$ ThT, $20 \mu \mathrm{g}$ recombinant a-syn synthesized in-house (see below), and 6 silica glass beads (OPS Diagnostics) in a total volume of $100 \mu \mathrm{l}$. Assay conditions were optimized using PD and healthy control brain lysates (Brain Bank Center Würzburg, BrainNet Europe Brain Bank Consortium Network). The 96 -well plates were incubated at $37^{\circ} \mathrm{C}$ with cycles of $1 \mathrm{~min}$ circular shaking $432 \mathrm{rpm}$ and $14 \mathrm{~min}$ rest with fluorescence readings every $45 \mathrm{~min}$ in a Tecan Infinite M200 microplate reader (Tecan Group Ltd., Switzerland). Raw data were normalized to a percentage of the maximal fluorescence response $(60,000 \mathrm{AU})$. The sample was considered to be positive if the fluorescence signal of at least 2 of the 4 replicates exceeded the $10 \%$ cut-off threshold. The assays were run for $60-80 \mathrm{~h}$.

\section{Recombinant a-syn protein expression}

An in-house synthesized recombinant a-syn protein was used in the Würzburg-based RT-QulC assay. A bacterial plasmid carrying human a-syn TAT mutant gene (kindly provided by Professor Roucou, University of Sherbrooke, Canada) was used to avoid cysteine misincorporation at codon 136 during bacterial expression and subsequent a-syn dimerization $^{46,47}$. The protein was overexpressed in BL21(DE3) Escherichia coli cells. Bacteria were grown at $37^{\circ} \mathrm{C}$ to $\mathrm{OD}_{600 \mathrm{~nm}}$ of 0.7 in $\mathrm{LB}$ media, before the expression was induced for $2 \mathrm{~h}$ upon addition of $0.44 \mathrm{mM}$ isopropyl $\beta$-D-1thiogalactopyranoside. The bacterial pellets were resuspended in lysis buffer containing $300 \mathrm{mM} \mathrm{NaCl}, 50 \mathrm{mM}$ sodium dihydrogenphosphate $(\mathrm{pH}$ 7.4), $1 \mathrm{mM}$ phenylmethylsulfonyl fluoride, $0.1 \mathrm{mM}$ tris-(2-carboxyethyl) phosphine (TCEP), and $1 \mathrm{mg} / \mathrm{ml}$ lysozyme. Cells were lysed by sonication and the lysate was cleared by centrifugation for $30 \mathrm{~min}$ at $38,000 \times 9,4^{\circ} \mathrm{C}$. The cleared lysate was mixed with Ni-NTA agarose (Qiagen) and incubated overnight at $4{ }^{\circ} \mathrm{C}$. Ni-NTA beads were transferred into a gravity flow chromatography column, washed with 40 volumes of lysis buffer. The protein was eluted with $125 \mathrm{mM} \mathrm{NaCl}, 300 \mathrm{mM}$ imidazole, $0.1 \mathrm{mM}$ TCEP, and $25 \mathrm{mM}$ sodium dihydrogenphosphate (pH 7.4). Roughly $15 \mathrm{mg}$ recombinant a-syn were purified from 1 liter of expression culture. Fractions containing the target protein were pooled and further purified by size exclusion chromatography (HiLoad Superdex 75 16/600 pg, Cytiva, USA) in PBS ( $\mathrm{pH}$ 7.4). Prior to long-term storage at $-80^{\circ} \mathrm{C}$, the protein was diluted to $5 \mathrm{mg} / \mathrm{ml}$ and flash-frozen in liquid nitrogen.

\section{Parameters of RT-QuIC seeding activity and skin RT-QuIC score}

In addition to the above described positive/negative outcome for each sample, the following parameters were calculated: mean ThT fluorescence at the end of 50-h (Cleveland) or 70-h (Würzburg) runs, referred to as final percentage of ThT fluorescence, the time (h) for a reaction to reach $50 \%$ of the maximum fluorescence (T50), and duration of lag phase (the reaction time [h] required to cross the fluorescence threshold). Assays with subthreshold signals were assigned T50 and lag phase equal to the duration of RT-QulC runs ( $\geq 50 \mathrm{~h}$ ).

To characterize the validity of a-syn seeding activity across multiple experiments and skin biopsy sites, a unified skin score of a-syn seeding activity was calculated. The rate of positive replicates out of four replicates per sample in both Cleveland and Würzburg assays was calculated for each biopsy site (i.e., $4 / 4=1,3 / 4=0.75,2 / 4=0.5,1 / 4=0.25$, and $0 / 4=0$ ), as was similarly described in previous studies ${ }^{17,48}$. The rate of positive replicates was then averaged for each subject across all biopsy sites resulting in a laboratory-specific score for each participant, ranging from 0 to 1 . The scores positively correlated with the mean final percentage of ThT fluorescence as a direct parameter of RT-QulC seeding activity (Spearman's $\rho=0.79, p<0.001$ ). The average of the laboratory-specific scores is further referred to as the "skin RT-QuIC score." A score of at least 0.5 (similarly to conventionally used 2 out of 4 replicates) was considered clearly positive, and a score of $\leq 0.25$ was considered negative. An intermediate score between 0.25 and 0.5 was included to account for a possible uneven distribution of a-syn seeding activity across biopsy sites.

\section{Statistical analysis}

Statistical analyses were conducted using the $\mathrm{R}$ software (RStudio version 1.3.1073; Boston, MA, USA) ${ }^{49}$ and GraphPad Prism version 9.0.0 (San Diego, CA, USA). For intergroup comparisons of normally distributed data (such as percentage of ThT fluorescence), an unpaired two-sided $t$ test was used and data were represented by means and standard deviations. Fisher's exact test was used for categorical data. In the case of subgroup comparisons involving the skin RT-QulC score, two-sided Mann-Whitney $U$-Test was applied, and data were illustrated by medians and interquartile ranges. To correlate skin RT-QulC score with numerical clinical parameters, such as NMSS or MoCA, Spearman's $\rho$ correlation coefficient was applied. For inter-laboratory reproducibility estimations, Cohen's kappa coefficients calculated using "kappa2" function of the "irr" library and Spearman's $\rho$ correlation coefficient using $\mathrm{R}$ were applied. Figures were created in ggplot2 version 3.3.2 and GraphPad.

\section{Reporting summary}

Further information on research design is available in the Nature Research Reporting Summary linked to this article.

\section{DATA AVAILABILITY}

The raw data that support the findings of this study are available from the corresponding authors upon reasonable request.

Received: 21 April 2021; Accepted: 21 October 2021; Published online: 15 November 2021

\section{REFERENCES}

1. Postuma, R. B. et al. MDS clinical diagnostic criteria for Parkinson's disease: MDSPD clinical diagnostic criteria. Mov. Disord. 30, 1591-1601 (2015).

2. Rizzo, G. et al. Accuracy of clinical diagnosis of Parkinson disease: a systematic review and meta-analysis. Neurology 86, 566-576 (2016).

3. Adler, C. H. et al. Submandibular gland needle biopsy for the diagnosis of Parkinson disease. Neurology 82, 858-864 (2014).

4. Lebouvier, T. et al. Pathological lesions in colonic biopsies during Parkinson's disease. Gut 57, 1741-1743 (2008).

5. Doppler, K. et al. Cutaneous neuropathy in Parkinson's disease: a window into brain pathology. Acta Neuropathol. 128, 99-109 (2014).

6. Donadio, V. et al. Skin nerve a-synuclein deposits: a biomarker for idiopathic Parkinson disease. Neurology 82, 1362-1369 (2014).

7. Donadio, V. et al. Abnormal a-synuclein deposits in skin nerves: intra- and interlaboratory reproducibility. Eur. J. Neurol. 26, 1245-1251 (2019).

8. Ikemura, M. et al. Lewy body pathology involves cutaneous nerves. J. Neuropathol. Exp. Neurol. 67, 945-953 (2008).

9. Kuzkina, A. et al. The aggregation state of a-synuclein deposits in dermal nerve fibers of patients with Parkinson's disease resembles that in the brain. Parkinsonism Relat. Disord. 64, 66-72 (2019).

10. Wang, N., Gibbons, C. H., Lafo, J. \& Freeman, R. a-Synuclein in cutaneous autonomic nerves. Neurology 81, 1604-1610 (2013).

11. Gibbons, C. H., Garcia, J., Wang, N., Shih, L. C. \& Freeman, R. The diagnostic discrimination of cutaneous a-synuclein deposition in Parkinson disease. Neurology 87, 505-512 (2016).

12. Chahine, L. M. et al. In vivo distribution of a-synuclein in multiple tissues and biofluids in Parkinson disease. Neurology 95, e1267-e1284 (2020).

13. Mazzetti, S. et al. a-Synuclein oligomers in skin biopsy of idiopathic and monozygotic twin patients with Parkinson's disease. Brain 143, 920-931 (2020).

14. Fairfoul, G. et al. Alpha-synuclein RT-QuIC in the CSF of patients with alphasynucleinopathies. Ann. Clin. Transl. Neurol. 3, 812-818 (2016).

15. Groveman, B. R. et al. Rapid and ultra-sensitive quantitation of disease-associated a-synuclein seeds in brain and cerebrospinal fluid by aSyn RT-QuIC. Acta Neuropathol. Commun. 6, 7 (2018).

16. Wang, Z. et al. Skin a-synuclein aggregation seeding activity as a novel biomarker for Parkinson disease. JAMA Neurol. 78, 1-11 (2021).

17. Cramm, M. et al. Stability and reproducibility underscore utility of RT-QulC for diagnosis of Creutzfeldt-Jakob disease. Mol. Neurobiol. 53, 1896-1904 (2016).

18. Wilham, J. M. et al. Rapid end-point quantitation of prion seeding activity with sensitivity comparable to bioassays. PLoS Pathog. 6, e1001217 (2010).

19. Doppler, K. et al. Dermal phospho-alpha-synuclein deposits confirm REM sleep behaviour disorder as prodromal Parkinson's disease. Acta Neuropathol. 133, 535-545 (2017).

20. Donadio, V. et al. Skin a-synuclein deposits differ in clinical variants of synucleinopathy: an in vivo study. Sci. Rep. 8, 14246 (2018).

21. Doppler, K. et al. Distinctive distribution of phospho-alpha-synuclein in dermal nerves in multiple system atrophy. Mov. Disord. 30, 1688-1692 (2015).

22. Brumberg, J. et al. Dermal and cardiac autonomic fiber involvement in Parkinson's disease and multiple system atrophy. Neurobiol. Dis. 153, 105332 (2021).

23. Haga, R. et al. Clinical utility of skin biopsy in differentiating between parkinson's disease and multiple system atrophy. Parkinsons Dis. 2015, 1-7 (2015).

24. Brännström, K. et al. A generic method for design of oligomer-specific antibodies. PLoS ONE 9, e90857 (2014). 
25. Shahnawaz, M. et al. Development of a biochemical diagnosis of Parkinson disease by detection of a-synuclein misfolded aggregates in cerebrospinal fluid. JAMA Neurol. 74, 163 (2017).

26. Kang, U. J. et al. Comparative study of cerebrospinal fluid a-synuclein seeding aggregation assays for diagnosis of Parkinson's disease. Mov. Disord. 34, 536-544 (2019).

27. Orrù, C. D. et al. A rapid a-synuclein seed assay of Parkinson's disease CSF panel shows high diagnostic accuracy. Ann. Clin. Transl. Neurol. 8, 374-384 (2021).

28. Tanei, Z.-I. et al. Lewy pathology of the esophagus correlates with the progression of Lewy body disease: a Japanese cohort study of autopsy cases. Acta Neuropathol. 141, 25-37 (2021).

29. Antelmi, E., Donadio, V., Incensi, A., Plazzi, G. \& Liguori, R. Skin nerve phosphorylated a-synuclein deposits in idiopathic REM sleep behavior disorder. Neurology 88, 2128-2131 (2017).

30. Al-Qassabi, A. et al. Immunohistochemical detection of synuclein pathology in skin in idiopathic rapid eye movement sleep behavior disorder and parkinsonism. Mov. Disord. 36, 895-904 (2021).

31. Postuma, R. B. et al. REM sleep behavior disorder and neuropathology in Parkinson's disease. Mov. Disord. 30, 1413-1417 (2015).

32. Adams-Carr, K. L. et al. Constipation preceding Parkinson's disease: a systematic review and meta-analysis. J. Neurol. Neurosurg. Psychiatry 87, 710-716 (2016).

33. Kong, W. L., Huang, Y., Qian, E. \& Morris, M. J. Constipation and sleep behaviour disorder associate with processing speed and attention in males with Parkinson's disease over five years follow-up. Sci. Rep. 10, 19014 (2020).

34. Ning, $\mathrm{H}$. et al. Baseline concentration of misfolded a-synuclein aggregates in cerebrospinal fluid predicts risk of cognitive decline in Parkinson's disease. Neuropathol. Appl. Neurobiol. 45, 398-409 (2019).

35. Fereshtehnejad, S.-M., Zeighami, Y., Dagher, A. \& Postuma, R. B. Clinical criteria for subtyping Parkinson's disease: biomarkers and longitudinal progression. Brain 140, 1959-1976 (2017).

36. Borghammer, P. \& Van Den Berge, N. Brain-first versus gut-first Parkinson's disease: a hypothesis. J. Parkinsons Dis. 9, S281-S295 (2019).

37. Poulopoulos, M., Levy, O. A. \& Alcalay, R. N. The neuropathology of genetic Parkinson's disease. Mov. Disord. 27, 831-842 (2012).

38. Takanashi, M., Li, Y. \& Hattori, N. Absence of Lewy pathology associated with PINK1 homozygous mutation. Neurology 86, 2212-2213 (2016).

39. Johansen, K. K., Jørgensen, J. V., White, L. R., Farrer, M. J. \& Aasly, J. O. Parkinsonrelated genetics in patients treated with deep brain stimulation: genetics in DBS patients. Acta Neurol. Scand. 123, 201-206 (2011).

40. Rossi, M. et al. Ultrasensitive RT-QulC assay with high sensitivity and specificity for Lewy body-associated synucleinopathies. Acta Neuropathol. 140, 49-62 (2020).

41. Shahnawaz, M. et al. Discriminating a-synuclein strains in Parkinson's disease and multiple system atrophy. Nature 578, 273-277 (2020).

42. Hoehn, M. M. \& Yahr, M. D. Parkinsonism: onset, progression and mortality. Neurology 17, 427-442 (1967).

43. Storch, A. et al. Non-motor Symptoms Questionnaire und Scale für das idiopathische Parkinson-Syndrom: interkulturell adaptierte versionen in deutscher sprache. Nervenarzt 81, 980-985 (2010).

44. Postuma, R. B. et al. A single-question screen for rapid eye movement sleep behavior disorder: a multicenter validation study. Mov. Disord. 27, 913-916 (2012).

45. Heinzel, S. et al. Update of the MDS research criteria for prodromal Parkinson's disease. Mov. Disord. 34, 1464-1470 (2019).

46. Roostaee, A., Beaudoin, S., Staskevicius, A. \& Roucou, X. Aggregation and neu rotoxicity of recombinant a-synuclein aggregates initiated by dimerization. Mol. Neurodegener. 8, 5 (2013).

47. Masuda, M. et al. Cysteine misincorporation in bacterially expressed human asynuclein. FEBS Lett. 580, 1775-1779 (2006).

48. Schmitz, M. et al. The real-time quaking-induced conversion assay for detection of human prion disease and study of other protein misfolding diseases. Nat. Protoc. 11, 2233-2242 (2016).

49. R Core Team. R: A Language and Environment for Statistical Computing (R Foundation for Statistical Computing, 2020).

\section{ACKNOWLEDGEMENTS}

We thank Professor Xavier Roucou, PhD and Jean-François Jacques, MSc for providing the $a$-syn plasmid. We are thankful to Dr. Maren Schubert and Dr. Lars Schönemann from the recombinant protein expression facility of the Rudolf-Virchow-Center for the expression and purification of a-syn. We thank Camelia-Maria Monoranu (Neuropathology Würzburg) for providing brain bank tissues. We are grateful to Antonia Kohl for excellent technical assistance. A.K. was supported by a Clinician-Scientist Scholarship from the Interdisciplinary Center for Clinical Research (IZKF). The study performed at the Cleveland center was supported by the American Parkinson Disease Association (to S.G.C.), the US National Institutes of Health (NIH)/National Institute on Aging (R01AG061388 to S.G.C.), and NIH/National Institute of Neurological Disorders and Stroke (U01NS112010 to W.-Q.Z., S.A.G., and S.G.C. and R01NS118760 to S.G.C.)

\section{AUTHOR CONTRIBUTIONS}

A.K., J.V., C.S., K.D. and S.G.C. contributed to the conception and design of the study A.K., C.B., D.S., J.R., W.W., A.-L.S., C.T., S.A.G., W.-Q.Z., K.D. and S.G.C. contributed to the acquisition and analysis of data. A.K., C.B., K.D. and S.G.C. contributed to drafting of the manuscript and preparation of figures. All authors contributed to revising the manuscript, approved the completed version, and agree to be accountable for all aspects of the work in ensuring that questions related to the accuracy or integrity of any part of the work are appropriately investigated and resolved.

\section{FUNDING}

Open Access funding enabled and organized by Projekt DEAL. This publication was supported by the Open Access Publication Fund of the University of Wuerzburg.

\section{COMPETING INTERESTS}

The authors declare no competing interests.

\section{ADDITIONAL INFORMATION}

Supplementary information The online version contains supplementary material available at https://doi.org/10.1038/s41531-021-00242-2.

Correspondence and requests for materials should be addressed to Kathrin Doppler or Shu G. Chen.

Reprints and permission information is available at http://www.nature.com/ reprints

Publisher's note Springer Nature remains neutral with regard to jurisdictional claims in published maps and institutional affiliations.

Open Access This article is licensed under a Creative Commons Attribution 4.0 International License, which permits use, sharing, adaptation, distribution and reproduction in any medium or format, as long as you give appropriate credit to the original author(s) and the source, provide a link to the Creative Commons license, and indicate if changes were made. The images or other third party material in this article are included in the article's Creative Commons license, unless indicated otherwise in a credit line to the material. If material is not included in the article's Creative Commons license and your intended use is not permitted by statutory regulation or exceeds the permitted use, you will need to obtain permission directly from the copyright holder. To view a copy of this license, visit http://creativecommons. org/licenses/by/4.0/.

(c) The Author(s) 2021 